\title{
SMS Notifying Design in Intra-Day Scheduling Security Check System
}

\author{
Cui-Hui Yan', Licheng Sha ${ }^{2}$, Sai Dai', Hai-Dong Zhao' \\ ${ }^{1}$ Power System Automation Department, China Electric Power Research Institute, Beijing \\ ${ }^{2}$ State Grid Beijing Electric Power Company, Beijing \\ Email: yancuihui2008@126.com,421773717@qq.com
}

How to cite this paper: Yan, C.-H., Sha, L.C., Dai, S. and Zhao, H.-D. (2017) SMS Notifying Design in Intra-Day Scheduling Security Check System. Energy and Power Engineering, 9, 535-543. https://doi.org/10.4236/epe.2017.94B059

Received: February 15, 2017

Accepted: March 30, 2017

Published: April 6, 2017

\begin{abstract}
In order to master the future operation and stability of power grid exactly, and gasp the weak point accurately, the requirement of power data quality become strict, and the data timeliness of power gird change into outstandingly more and more, because of this, in this paper propose the SMS notifying method of intra-day scheduling data based on safely data principle. The principle is mainly complied with the data source existed or not, the data is coincident to the power grid model, the data is unbroken or not and it is reasonable with the physical reality, then it can obtain better convergence and reasonable intra-day check power data. In order to accelerate the information and network pace of the power grid, the SMS notifying can monitoring data quality without time delay. It dredge the vast path for the future power market into use with the wide range, then, can more effective to ensure the convergence and accuracy of safe check calculation, it provides an effective guarantee with the safe and stable operation of the power grid, in the same way, it is also an efficient method to provides effective guarantee for power grid safe operation from the data source.
\end{abstract}

\section{Keywords}

Security Checking, Intra-Day Scheduling, SMS Notifying

\section{Introduction}

Following with the voltage grade rise and power grid structure becoming complicated more and more, the electrical contact of the whole grid compactness increased remarkable, the grid shape and properties are confronted with profound changes, the safe and stable level of the power grid mutually restricted with each other. Grid structure and the way of power flow change is much large, new energy sources such as wind power, photovoltaic large-scale access further ag- 
gravated the uncertainty in the power grid operation mode as in [1] and [2]. The security and stability level of power grid ascend to a new height, and the challenge of power grid security analysis and evaluation analysis become tremendous.

Security check is one of the application of the smart gird scheduling control system, it is much more important among those applications, it can guarantee the grid operating safely and steady, it also can provide check service for the scheduling plan and power grid operation [3] (such as operation order, temporary operation).

In order to adapt the model expansion of power grid and the more complex structure, the research of security check is much more rich and colorful. For the sake of power market, it provides a necessary platform and technical support. Paper [4] is proposed a grid computing method of scheduling plan security check based on SOA (Service Oriented Architecture), it also analysis the security check section adopted the parallel strategy quickly. Many type of security check with different range [5] of three level day-ahead generations scheduling from the state to the province are briefly reviewed. Power flow data generation based on similar day [6] selection is proposed to improve the accuracy and fineness of security check for scheduling plans. It is provide customizable security check service for day-ahead scheduling [7]. A set of database with check rule can used for scheduling plan data [12], which according to the scheduling plan data characteristics and requirements, it check the data quality inserted into the database which in consideration of the system function balance, unit operation constrains, data completeness and rationality multiple aspects. In this paper, it is designed the SMS notifying method of scheduling plan data quality without time delay to ensure the safe check calculation effectively and orderly conducted, which rely on the smart power grid construction boost and data quality optimized of the grid big data. The messages notifying platform design is relatively mature [8], and the application in electric power grid has achieved good results [9], but the application of short message service (SMS) notifying in safe check with data quality is scarce. The safe check calculation convergence or not is mainly depend on the data quality, better grasp the data quality of scheduling plan is the key point, it can better analysis the security and stability of the power system, and realize possible weak part in the power system, therefore, the evaluation analysis indicators of safe check system [13] has more practical significance. In consequence, the SMS notifying of basic data can make the data quality control becoming more timeless, more quickly and convenient. It can effectively promote the network process of the power grid, which is more important for the future power market to carry out.

\section{Message Warning Platform Presentation}

The platform developed according to the SMS protocol, which can send message to the users belong to mobile, Unicom, SMS operators and it can also receive massage via cmpp3.0, SGIP1.2, SMGP3.0 protocol, it can also send massage with 
flock. The massage send function can meet the requirements of all kinds of business system, it can also connect with other business system through dynamic link libraries, TCP/IP, HTTP database stored procedures, and it can support secondary development based on the platform by VC, c++, VB, DELPHI, PB, JAVA, COM, J2EE, et instrument [10].

The message waring platform installed one SMS hardware gateway, and one software IP interface gateway. First of all, the primary task is entering the system as an administrator to register the application of SMS massage waring, and then fill in the important information fields of message mechanism, and open the message warning application channel.

Message machine is applied MySQL database which is much popular now. MySQL is a smaller relational database with open source of database management system, the developers is the Swedish MySQL AB Company, the database is currently widely used in small and medium-sized websites on Internet. A relational database consists of one or more tables. The SQL language is one of the standardization of language to access the database. The message waring system connected to the message waring service station through the message bus, and then send message to users through message send server. The description of message waring topology tree is proposed here as shown in Figure 1.

\section{Intra-Day Scheduling Security Check}

\subsection{Intra-Day Scheduling Security Check Presentation}

Security check need to obtain a suitable model of the grid, using grid mode data and the scheduling date, which including the bus load forecasting, generation schedule, contact line plan, maintenance plan and direct current transmission plans to form a computational model, for variety of monitoring objects, made security analysis in the case of many expected failures, the analysis including trends analysis, sensitivity analysis, static security analysis, and pointing out the weak parts of the power grid.

Intra-day scheduling security check is similar to security check, their process is the same, but the input data is different in the time scale. Intra-day scheduling

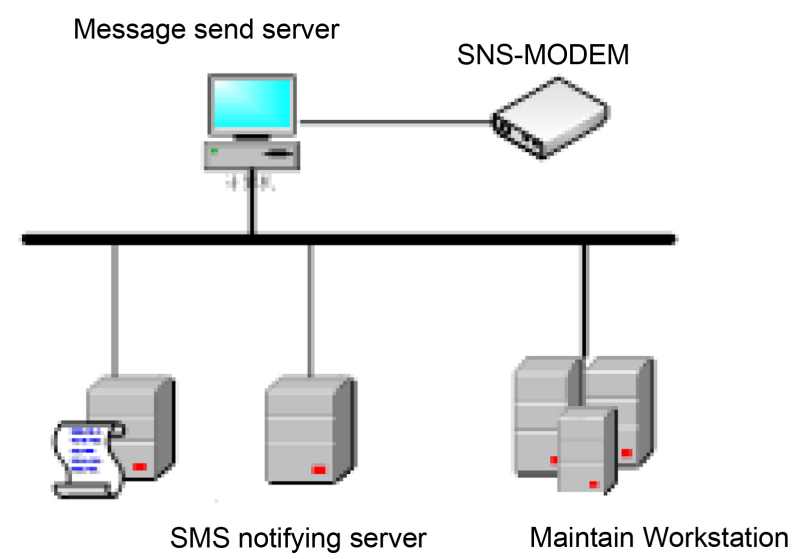

Figure 1. SMS notify system topology. 
check is plan to analysis the security situation of the power system of intra-day scheduling plan, evaluate power grid risk point and weak link of power grid in the future operation time and check the reasonability of intra-day scheduling plan. At the same time, the check time point and amounts also can be selected (the time scale is the next time of 15 minutes and within 4 hours any time parts) through man-machine interface, and then submit security check calculation task to server, it can conduct the security check of specified period with intra-day scheduling plan, count and analysis the power gird operation dangerous points and the over limited power flow results within the 16 time parts in the future 4 hours. Intra-day scheduling plan check operated according to intra-day scheduling plan, ultra short-term load forecast and power grid network online operation data to generate the gird power flow of future time period automatically, it is taking a full account of scheduling plan data characteristics and arrangement principles of operation, it can carry out initial state power flow and N-1 static security analysis, it can judge the power equipment power flow over the limited value under the scheduling mode or not, then give out the waring information.

\subsection{Intra-Day Scheduling Overall Architecture}

The intra-day scheduling check application is based on the smart grid scheduling technology support system platform, it can connected with EMS, OPS, OMS application through the base platform [11] and [15], then can acquire power grid basic data such as scheduling plan and power gird on line operation mode data, it can achieve the crosswise data share and also the other services. The intra-day scheduling plan check is composed of basic data and calculation module, basic module is to realize process control, data dispose, security check results display function, calculation module is mainly to realize the future power flow calculation and static security analysis of the core computing functions.

\subsection{Intra-Day Scheduling Check Power Flow Calculation Process}

The data source of intra-day scheduling plan check is multiple, and has data interaction and communication multiple applications embedded in the platform, and the support of platform with power grid network data, then can realize the system scheduling plan check goal through initial state power flow calculation and safety analysis calculation, building on this can obtain scheduling plan conformed to the requirements of the power grid dispatching and ensured the power gird safety operation, the intra-day scheduling plan check function is as follows:

1) Data preparation

The data used here are intra-day exchange plan, intra-day generation plan, intra-day equipment stop arrange plan, load forecast scheduling plan and realtime operation model of power grid which can obtained from data platform coming from other applications, the data is the same with the day-ahead scheduling plan, the accuracy of intra-day scheduling plan is directly related to the right and the integrity of data acquisition. Therefore, the data check is necessary. 


\section{2) Data check}

The intra-day plan source is multiple, and in order to ensure the effectiveness of scheduling plan check using the automatic checking method, the check is mainly carried out on the integrity and logic contrasted with power grid operation with each type scheduling plan, the check results can quickly catch the data problem and assist the dispatcher adjusting the data subsequently.

3) Function Configuration

The intra-day scheduling security check has two model such as automatic control and manual control, therefore, the process can be intervened by human according to the flexible configuration module based on the process, the flexible configuration is consisted of computing time part, check content, calculation scope of the intra-day scheduling check, it can also start, pause, continue and stop with freedom.

4) Check calculation

The intra-day scheduling security check is including the future state power flow and the static state security analysis. The future state power flow analysis of intra-day scheduling security check is power flow actually, the data sources is coming from other applications, the mainly sources are power gird model, system load forecast, busload forecast, equipment operation-stop scheduling (equipment operation model changes, etc), generation scheduling, exchanging plan and the power grid surface, etc. then can estimate single device and stable sections overload or exceed its limit. The corresponding flow function is as follows:

$$
\left\{\begin{array}{l}
P_{i}=U_{i} \sum_{j \in i} U_{j}\left(G_{i j} \cos \theta_{i j}+B_{i j} \sin \theta_{i j}\right) \\
Q_{i}=U_{i} \sum_{j \in i} U_{j}\left(G_{i j} \sin \theta_{i j}+B_{i j} \cos \theta_{i j}\right)
\end{array}\right.
$$

That: $P_{i}$ and $Q_{i}$ is the active power and reactive power of spot $i ; j \in i$ denote spots who connected with spot $i ; U_{i}$ and $U_{j}$ is the voltage of spot $i$ and $j$ respectively; $G_{i j}$ and $B_{i j}$ are elements of spot matrix; $Q_{i j}$ is phase angle difference between spot $i$ and $j$.

According to the gird model and equipment stop scheduling can determine the topology of the check section, then the element $G_{i j}$ and $B_{i j}$ can be determined,

According to the data of other nodes bus load and power generation plan can determine the data $P_{i}$ and $Q_{i}$, then according to flow equations to calculate the node voltage and phase, the scheduling flow calculation is different from the general power flow calculation, there is existed errors between calculation, because of the lack of reactive power flow in the scheduling data, so the calculation is more complex. The quality of data requirements are more stringent, the not convergence condition appeared in the calculation is also more timely treatment, when cannot find the reasons for convergence, data quality is the primary guarantee. Intra-day scheduling is different from the day-ahead scheduling, which has imminently request in time scale, it mainly calculate the next four hours from now, therefore, the time required to calculate the more urgent. More atten- 
tions to the accuracy of power flow results.

Through data validation and model splicing, obtained the latest model of the power grid, after power flow calculation, it can obtain the state power flow distribution in transmission lines and transformers and other equipment, so can analysis the power grid initial state operation condition, the power flow is the primary means of power system analysis, $\mathrm{N}-1$ is the basement of power grid safety analysis.

\subsection{Security Analysis in Check}

The load flow calculation is applied in the security check, and the safety analysis calculation is also taken into account, the security analysis in here only consider the safety of the steady-state operation after the accident, never considered the dynamic transfer of the operation status in the accident process [14], the expected fault is generally the breaking failure, which is determined by the situation of the grid and the experience of the dispatchers. The expected failure includes branch breaking and engine breaking. The algorithm mainly used in the safety analysis is the distribution coefficient method.

The intension of distribution coefficient method first is solving the distribution coefficient with a slip road off relate to the other slip, then the current of slip breaking multiplied by the corresponding distribution coefficient converted to calculate the slip. The distribution factor function is calculated as follows:

$$
L_{i, j-k, m}=\frac{x_{k, m}\left(X_{i, k}-X_{i, m}-X_{j, k}+X_{j, m}\right)}{x_{i, j}\left(x_{k, m}-X_{k, k}-X_{m, m}+2 X_{k, m}\right)}
$$

That, $L_{i, j-k, m}$ the slip km's current change which is caused in slip ij when a unit current through slip km after breaking, $x_{i, j}$ is the reactance value of slip $i j, x_{k, m}$ is the reactance value of slip $\mathrm{km}, x_{i, k}$ is the element withi row and $\mathrm{k}$ column in the impedance matrix (which is mutual reactance), $x_{k, k}$ is the element with $\mathrm{k}$ row and $\mathrm{k}$ column in the impedance matrix (which is self-reactance)

\section{SMS Notifying Method in Intra-Day Data Check}

\subsection{SMS Notifying File Produce}

In order to check the accuracy of the calculation and the convergence of the power flow calculation, the data source of intra-day scheduling is large, it is essential with the accuracy of the check calculation and the convergence of the power flow calculation. The acquired seven categories data should be checked accordance to the check requirements, it must checking each data source according to data check principle, and then generate an error message file in every 15 minutes, the file name can be accurate to the point of time (format: YYYYMM-DD-HHHH) given in order to distinguish between different date and time of the file. Error messages are subject to the following principles:

- Existence of the data source: the exchange of the day, the day of the power generation plan, the date of equipment maintenance program, load forecasting data, such as the existence of the source file, if not exist alarm; 
- Consistency: the exchange scheduling, power generation scheduling, equipment maintenance scheduling, load forecast, the equipment name in the stable section member are consistent with the equipment name of the basic model.

- Integrity: on the one hand refers to the device in the power grid is complete or not, bus load forecasting, power generation plan must covering all equipment scheduling within the scope of section members defined; on the other hand is refers to the integrity of the scheduling periods, whether the specified time moment is to the next 4 hours (16 points) check time data.

- Logicality: the unit power generation plan does not exceed its maximum technical power output, the heating power generation unit to meet its requirements of the heating upper and lower limits.

\subsection{SMS File Parse}

In accordance with the above data verification principle, it is generated the error information writing in error information file, the local application will back-up an error message file for inspection. The error message file is transferred to the specified directory of the two areas of the intra-day application plan, and the file is transferred to the specified directory of the system's short message by the isolation device, and the process is completed in real time. Then, the error message file will be parsed, using MySQL storage program put the error information storage to MySQL database of the SMS machine, through the network to send SMS text messages to the specified users. SMS notifying logic structure is shown in-

\section{Figure 2.}

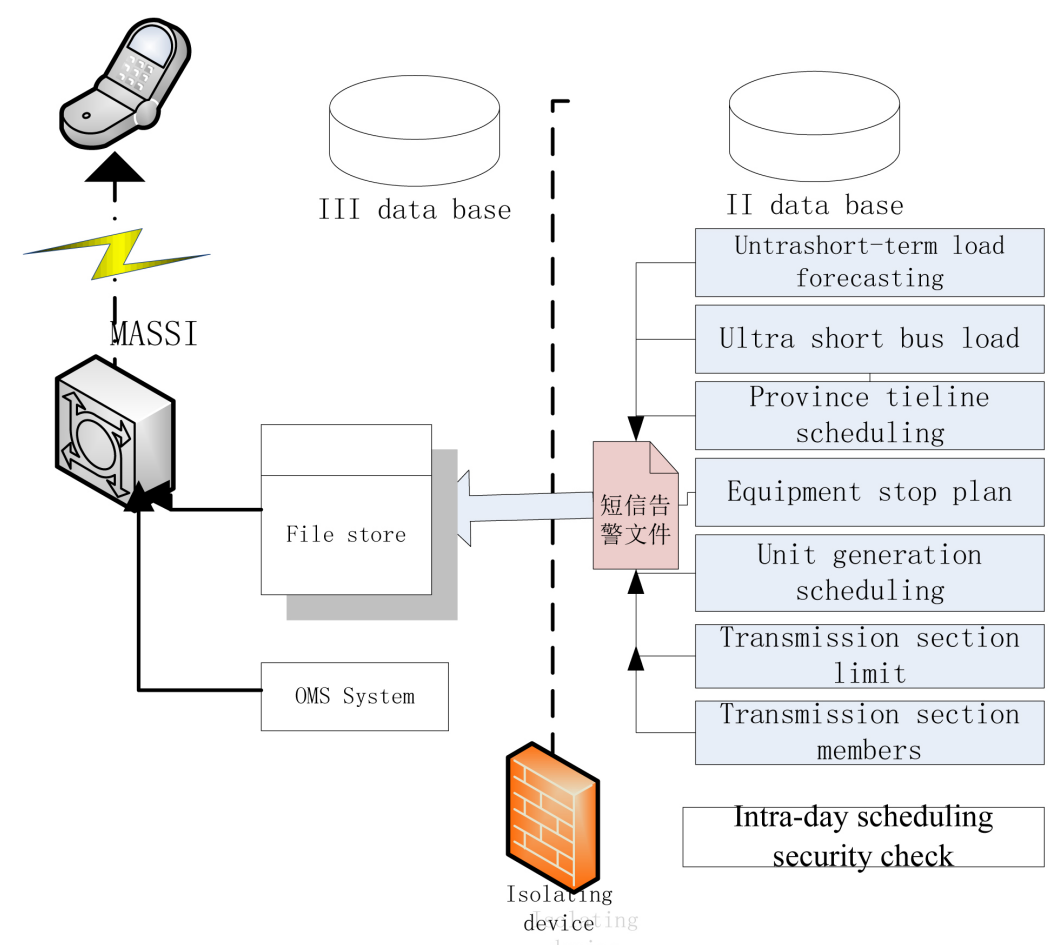

Figure 2. SMS notifying logic diagram. 


\section{Conclusion}

Based on the results of safety check calculation, it can be used to analysis the overload risk of line and transformers. It's effectively demonstrated the weak link and severity of the power grid. In order to ensure the convergence and accuracy of the scheduling check, the data quality is controlled without time delay before scheduling check, which provides a complete and reasonable data source for the analysis and display of the results. Power market of UHV and cloud scheduling control system are involved into the existing system, which put forward higher requirements on the grid management, the quality of data cannot be ignored, it need to control data on real-time, a good grasp of the data, by means of electronic system of the existing achievements, the electric power data through communication network and modernization, also can be the popularity of grid applications to achieve a full range of network information, digitalization and information, real-time control of network security, it become the security assistant safety for the safe operation of power grid. For the safety and stability of the operation of large power grid have a more effective means and the way forward for the future.

\section{References}

[1] Gao, Y.J., Zhang, Y.K., Li, R.R., et al. (2015)MaintenanceReserve Capacity Evaluation and its Application Considering Wind Power and Maintenance Demand Uncertainty. High Voltage Apparatus, 51, 31-38.

[2] Hou, Y.Q., Li, W. (2013) Influence of Integration Of Large-Scale Photovoltaic On Grid And Key Factors Restricting Tis Accepted Capacity.Power System and Clean Energy, 29,73-77.

[3] Zhang, Z.G. and Xia, Q. (2009)Architecture and Key Technologies for Generation Scheduling of Smart Grid. Power System Technology,33, 1-8.

[4] Wang, X.Z., Yan, Z. and Shen, C., Li, N.H., Jing, L. and Li, H.J. (2011)Grid Computing of operation Scheduling with Security Constraint Based on SOA. Power System Protection and Control,39, 90-95.

[5] Lu, G.P. (2014)State Center and Province Three rank of Day-ahead Generating Statics Security Check. Sichuan Electric Power Technology,37,66-69.

[6] Zhou, H.F., Xu, W., Bao, Y.H., Guo, J., Yan, M.H. and Wang, M.X. (2015)Power Flow data Generation of Security Check for Scheduling Plans based on Similar Day selection. Power System Protection and Control, 43, 87-93.

[7] Lu, Y., Lu, G.M., Yang, J.F.,et al.(2015)Security CheckService and Practical Technique Based on Smart GirdDispatching and Control System. Automation of Electric Power System, 39,171-176.

[8] Jin, J., Wang, F., Han, Q.X. and Wu, J. (2009) Development and Application of Short Messages Alarm Software in Qinghai Automation System. 28.

[9] Song, J.B. (2008)SMS Alarm Monitoring and Storage System of Electric Power Network Data. Master Thesis, 2008.

[10] Zhang, C.G. (2008)Design and Implementation of SMS Platform System. Master Thesis, 2008

[11] Xu, T., Yu, Y.J., Qian, Y.M. (2006) Security Correction Design on Generation Plan in Energy Management System. 30, 88-92. 
[12] Chang, L., Liu, Y.J., Zhu, C. and Cheng,L. (2012) A Rule-based Data Valldation Mechanism of power Scheduling.Automation of Electric Power System, 36, 98-102.

[13] Yan, C.H., Dai, S., Cheng X., Zhang, C.C,etc. (2012)Application and Research of Static Security Check in FU-jianPower System. 2012 International Conference on Electronics Information and Electrical Engineering (EIEE2012),11, 38-41.

[14] Hu, S.J., Li, D.M., Chen, Z.Y., et al.(2005)Security Checking and Optimal Adjustment system for Daily Generation Scheduling.Modern Electric Power, 22, 61-64.

[15] Xu, Tian, Yu, Y.J., Qian, Y.M. (2006)Security Correction Design on Generation plan in Energy Management System.Automation of Electric Power System, 30, 88-92.

Submit or recommend next manuscript to SCIRP and we will provide best service for you:

Accepting pre-submission inquiries through Email, Facebook, LinkedIn, Twitter, etc. A wide selection of journals (inclusive of 9 subjects, more than 200 journals)

Providing 24-hour high-quality service

User-friendly online submission system

Fair and swift peer-review system

Efficient typesetting and proofreading procedure

Display of the result of downloads and visits, as well as the number of cited articles

Maximum dissemination of your research work

Submit your manuscript at: http://papersubmission.scirp.org/

Or contact epe@scirp.org 